Received : July 25th 2017; Accepted : August $9^{\text {th }} 2017$; Avalaible Online : September $5^{\text {th }} 2016$

\title{
PERFORMANCE COMPARISON OF RULE GENERATION METHOD SUBSTRACTIVE CLUSTERING AND FUZZY C-MEANS CLUSTERING ON SUGENO'S INFERENCE FOR STROKE RISK DETECTION
}

\author{
Rekyan Regasari MP, Edy Santoso
}

\begin{abstract}
Fuzzy Inference is one method that can solve the problem of uncertainty in a decision-making or classification well. In inference, fuzzy rules that represent the need of expert knowledge in the relevant fields, so that the classification given decision or be appropriate expert knowledge. However there are times when experts are less able to represent the rules of the appropriate knowledge or knowledge that there is need of too many rules, so we need a method that can generate rules based on the data given expert.

At issue troke s disease risk detection, it also occurs because of the research that has been done by taking the direct rule of experts, it turns out less than the maximum accuracy, still $82.89 \%$. Substractive methods Clustering and Fuzzy C-Means (FCM) could generate rules by grouping algorithm, in which the existing training data are grouped in common and the rules of the group raised. Differences in the two methods are in determining the center of the cluster and assign each incoming data which groups.

Based on research that has been done, substractive average Clustering membrika better accuracy is $84.46 \%$, while $73.81 \%$ FCM. However, in the processing time FCM faster at 16.75 seconds to give an average processing time of 13:02 seconds.
\end{abstract}

Keywords: Stroke, substractive Clustering, Fuzzy CMeans

\section{Preliminary}

Fuzzy Inference System is one of the alternatives which can be used to resolve the issue of diagnosis or risk analysis. In this case, particularly regarding the detection of the risk of stroke. Fuzzy logic is the logic that has a value of vagueness or ambiguity that is used for reasoning [5]. For the reasoning necessary inference rules that come from an expert. But often an expert can not express explicit knowledge [3]. Previous research associated fuzzy inference system in case of early detection of the risk of stroke using a fuzzy inference system Mamdani method which rules are derived from an expert. The study resulted in the level of accuracy of $82.98 \%$ with the highest error potential by $23.08 \%$ [8]. Mamdani method is more like the human mind (an expert). But sometimes rules are obtained from experts is less than optimal when used as a fuzzy inference [9]. Therefore we need a medium of learning in generating rules. Rules that came from experts can be replaced by using clustering algorithms like FCM, k-means, subtractive clustering and nearest neighborhood clustering [2]. From the cluster is formed, will be analyzed as to what characteristics of the data in the cluster, so that can know what the variables that affect the level of risk of stroke in the cluster. The number of rules generated will equal the number of clusters [5].

Previously had done some research on the generation of rules. Among them by Ely Ratna Sayekti in 2013. In that study used fuzzy c-means as a medium of learning in generating rules. Rules resulting from the fuzzy cmeans, in turn, used to perform the grouping level of risk of breast cancer using Sugeno fuzzy inference systems order-one. From the results, the highest accuracy of $87 \%$ [7]. Substractive clestering also been applied in a variety of research with accuracy results vary between $80 \%$ to $93 \%$ [7].

This study will compare fuzzy clustering technique for generating rule, namely subtractive clustering and fuzzy c-means clustering. Substractive clustering method is a clustering algorithm based on the size of the potential of the data points in a variable. The point with the highest potential will be the first cluster center [2]. All the data points within the radius (the radius) is then removed to determine the next cluster. This process is repeated for all of the data is within a radius of 3 [4]. While fuzzy c-means is an adaptation of the k-means algorithm with smooth membership functions. Fuzzy cmeans enabling a data point to be a part for all centers [1]. So from that, the fuzzy c-means can be used to generate fuzzy rules from a set of data.

Rules generated by substractive clustering and fuzzy c-means and then used for the early detection of the risk of stroke using fuzzy inference systems. Fuzzy inference system used is the first order Sugeno. Selection of first order Sugeno method due to the consequent (output) in the form of a collection of constants [5]. As for calculating the degree of membership in each role can use Gauss function with the help of cluster center value and standard deviation [5].

\section{Stroke}

Risk factors for stroke is a medical condition or illness that existed at someone who is at risk of stroke. If this condition is not immediately controlled, it can 
make things worse and can lead to narrowing or rupture of blood vessels of the brain [10].

There are two kinds of stroke risk factors, the risk factors that can controlled and the risk factors can not controlled. Risk factors that can not controlled include:

1. Age.

Strokes can occur at any age, even children will remain a stroke more common in people who have aged (old). Each additional 10 years after age 55, there is an increased risk of stroke as much as twofold.

2. Gender

Stroke is more likely in men than in women. However, more than half the total stroke deaths occur in women. The use of birth control pills and pregnancy increases the risk of stroke for women.

3. Race

Deaths due to stroke is more common in AfricanAmericans than in whites. This is because they have a higher risk of suffering from high blood pressure, diabetes, and obesity.

While based on JNC 7 (Seventh Report of the Joint National Committee on Prevention, Detection, Evaluation, and Treatment of High Blood Pressure risk factors that can be changed / controlled include:

1. Blood pressure

The following is a classification of blood pressure.

Table 1 Classification of Blood Pressure

\begin{tabular}{|c|l|}
\hline Range & \multicolumn{1}{|c|}{ Information } \\
\hline$<120$ & Normal \\
$120-139$ & prehypertension \\
$140-159$ & Hypertension Stage I \\
$>160$ & Hypertension Phase II \\
\hline
\end{tabular}

2. Blood Sugar Levels

Here is a table of classification of blood sugar levels.

Table 2 Classification of Blood Sugar Levels

\begin{tabular}{|c|l|}
\hline Range & \multicolumn{1}{|c|}{ Information } \\
\hline$<60 \mathrm{mg} / \mathrm{dl}$ & Low \\
$60-130 \mathrm{mg} / \mathrm{dl}$ & Normal \\
$140-199 \mathrm{mg} / \mathrm{dl}$ & Intermediete \\
$>=200 \mathrm{mg} / \mathrm{dl}$ & Diabetes \\
\hline
\end{tabular}

3. Total Cholesterol Levels

The following is a classification of total cholesterol in the body.

Table 3 Classification Total Cholesterol Levels

\begin{tabular}{|c|l|}
\hline \multicolumn{1}{|c|}{ Range } & \multicolumn{1}{c|}{ Information } \\
\hline$<200 \mathrm{mg} / \mathrm{dl}$ & Normal \\
$200-239 \mathrm{mg} / \mathrm{dl}$ & High \\
$>=240 \mathrm{mg} / \mathrm{dl}$ & Very high \\
\hline
\end{tabular}

4. Low Density Lipoprotein (LDL)

The following is a classification of levels of Low Density Lipoprotein (LDL) in the human body.

Table 4 Classification of LDL

\begin{tabular}{|c|l|}
\hline \multicolumn{1}{|c|}{ Range } & \multicolumn{1}{c|}{ Information } \\
\hline$<100 \mathrm{mg} / \mathrm{dl}$ & Normal \\
$130-189 \mathrm{mg} / \mathrm{dl}$ & High \\
$>=190 \mathrm{mg} / \mathrm{dl}$ & Very high \\
\hline
\end{tabular}

\section{Uric acid}

Table 5 Classification of Uric Acid Levels Male

\begin{tabular}{|c|l|}
\hline Range & \multicolumn{1}{|c|}{ Information } \\
\hline$<=3.5 \mathrm{mg} / \mathrm{dl}$ & Low \\
$3.5 \mathrm{to} 7.0 \mathrm{mg} / \mathrm{dl}$ & Normal \\
$>=7.0 \mathrm{mg} / \mathrm{dl}$ & Uric acid \\
\hline
\end{tabular}

\section{BUN and creatinine}

7. Table 6 Classification of Women Uric Acid Levels

\begin{tabular}{|c|l|}
\hline Range & \multicolumn{1}{c|}{ Information } \\
\hline$<=2.6 \mathrm{mg} / \mathrm{dl}$ & Low \\
2.6 to $6.0 \mathrm{mg} / \mathrm{dl}$ & Normal \\
$>=6.0 \mathrm{mg} / \mathrm{dl}$ & Uric acid \\
\hline
\end{tabular}

Table 7 Classification BUN levels

\begin{tabular}{|c|l|}
\hline Range & \multicolumn{1}{|c|}{ Information } \\
\hline$<6 \mathrm{mg} / \mathrm{dl}$ & Low \\
$6-23 \mathrm{mg} / \mathrm{dl}$ & Normal \\
$>23 \mathrm{mg} / \mathrm{dl}$ & High \\
\hline
\end{tabular}

Table 8 Classification Creatinine

\begin{tabular}{|c|l|}
\hline Range & \multicolumn{1}{|c|}{ Information } \\
\hline$<0.7 \mathrm{mg} / \mathrm{dl}$ & Low \\
0.7 to $1.2 \mathrm{mg} / \mathrm{dl}$ & Normal \\
$>1.2 \mathrm{mg} / \mathrm{dl}$ & High \\
\hline
\end{tabular}

\section{III.Substractive Clustering}

Subtractive clustering is an unsupervised clustering algorithm to establish the number and cluster centers in accordance with the conditions of the data. The basic concept of the subtractive clustering is to determine the area in a variable that has a high density to the points in the vicinity. Point with the highest number of neighbors will be selected as the center of the cluster. The point will then be reduced density. Then the algorithm will select another point that has the highest neighbor to be the center of the next cluster. This will be done until all points tested [5]. If there are $\mathrm{N}$ pieces of data: X 1, X 2, ..., $\mathrm{Xn}$ and assuming that the data is already in a normal state, the density of point $\mathrm{X} \mathrm{k}$ can be calculated using Equation (1).

$$
D_{k}=\sum_{j=1}^{N}\left(-\frac{\|X k-X j\|}{\left(\frac{r}{2}\right)^{2}}\right)
$$

with $\mathrm{Xk}-\mathrm{Xj}$ is the distance between $\mathrm{Xk}$ to $\mathrm{Xj}$ and $\mathrm{r}$ is a positive constant which will then be known as fingers. Finger in the form of a vector that will determine how much influence the cluster center in each variable. Thus, a data point will have a density which is great if he has many close neighbors. After calculating the density of each point, then the point with the highest density will be chosen as the center of the cluster. Suppose $\mathrm{Xc1}$ is selected as the center point of the cluster, while the DC1 is a measure of density. Furthermore, the density of the point around will be reduced as in Equation (2). 
$D_{k}^{\prime}=D_{k}-D_{c 1} * \exp \left(-\frac{\left\|X_{k}-X_{c 1}\right\|}{\left(r_{\frac{b}{2}}\right)^{2}}\right)$

with rb is a positive constant. This means that the point the point which is close to the cluster center uc1 will experience a reduction in the density of large scale. This will lead to that point will be very difficult to be the center of the next cluster. $\mathrm{Rb}$ value indicates an environment that led to the point size of the reduced density. Rb usually worth more than the $r, r b=q *$ rb (usually squash_factor $(q)=1.25$ ).

Squash This factor used to multiply the value of the radius that determines the area around the center cluster. Once the density of each fixed point, then will find the center of the second cluster is $\mathrm{xC} 2$. After $\mathrm{xC} 2$ obtained, measure the density of each data point will be restored, and so on. On implementation, can be used two fractions as a differentiating factor, ie a ratio ccept and reject ratio. Both accept ratio and ratio reject both a valuable fractions of 0 to 1 . Accept ratio is a lower limit at which a data point that is a candidate (candidate) cluster centers are allowed to be the center of the cluster. The default value of the accept ratio of 0.5 . While the rejection ratio is the upper limit which a data point that is a candidate (candidate) cluster center is not allowed to be the center of the cluster. The default value of the reject ratio is 0.15 . In an iteration, if it has found a data point with the highest potential (eg Xk with potential Dk), will be followed by finding the ratio of potential data points with the highest potential of a data point in the early iterations (eg Xh with potential Dh). Quotient between Dk with Dh is then called by the ratio (ratio $=\mathrm{Dk} / \mathrm{Dh}$ ). There are three conditions that can occur in an iteration:

a. If the ratio> Accept ratio, the data point is accepted as a new cluster center.

b. When Reject ratio <ratio $\leq$ Accept ratio of the data points will be accepted as the center of a new cluster only if the data points are located at a considerable distance to the cluster center other (the sum between the ratio and the shortest distance data points to the center of the cluster more who have been there $\geq 1$ ). If the result of the sum of the ratio and the longest distance data points to the center of another cluster that already exist $<1$, then in addition to the data points will not be accepted as the center of the cluster, he already will not be considered again to be the center of a new cluster (potential is set equal to zero ).

c. If the ratio of $\leq$ Reject ratio, then there is no more data points to be considered to be candidates for the center of the cluster, the iteration is stopped.

\section{IV.Fuzzy C-Means}

Fuzzy c-means including a fuzzy clustering algorithm. This method was first introduced by Jim Bezdek in 1981. Fuzzy c-means clustering is a technique where each data point in a cluster is determined by the degree of membership. The basic concept of fuzzy c-means is to determine the location of the center of the cluster as a marker of the average for each cluster. By improving the center of the cluster and the degree of membership of each data point is repeated, it will be seen that the center of the cluster will move to the right location. This loop is based on the minimization of the objective function that describes the distance from a given data point toward the center cluster weighted by the degree of membership of the data points (Kusrini, 2010).

The output of the fuzzy c-means not a fuzzy inference system, melaikan a central row of the cluster and some degree of membership for each data point. This information can be used to construct a fuzzy inference system [6]. Fuzzy C-Means algorithm (FCM) is as follows [6]:

1. Input data will -cluster $X$ is a matrix of size $n x m$, where $n$ is the number of data samples and $m$ are the attributes of each data. $X_{i j}=$ The sample data to $\mathrm{i}(\mathrm{i}=1,2,3, \ldots, \mathrm{n})$, attribute $\mathrm{j}(\mathrm{j}=1,2,3, \ldots, \mathrm{m})$.

2. Determine:

The number of clusters $=\mathrm{c}$;

Rank $=\mathrm{w}$;

Maximum iterations $=$ MaxIter;

The smallest error Expected $=\xi$;

The objective function early $=\mathrm{P} 0=0$;

Early iterations $=\mathrm{t}=1$;

3. Generating random numbers $\mathrm{i}=1,2, \ldots, \mathrm{n} ; \mathrm{k}=1$, $2, \ldots, \mathrm{c}$; as elements of the initial partition matrix $\mathrm{U}$ with a range between 0 and 1 . Then, count the number of each column. The amount of each column of random numbers that are formed can be calculated using equation (3).

$$
Q_{i}=\sum_{k=1}^{c} \mu_{i k}
$$

with $\mathrm{j}=1,2, \ldots, \mathrm{n}$.

After summing each column of random numbers is formed, it then calculates the initial partition matrix initial partition matrix $U$. $U$ can be calculated by equation (4).

$$
\boldsymbol{\mu}_{i k}=\frac{\mu_{i k}}{Q_{i}}
$$

4. Calculating the k-th cluster center () based on the equation (5), with $\mathrm{k}=1,2, \ldots, \mathrm{c}$; and $\mathrm{j}=1,2, \ldots, \mathrm{m}$

$$
V_{k j}=\frac{\sum_{i=1}^{n}\left(\left(\mu_{i k}\right)^{w} * X_{i j}\right)}{\sum_{i=1}^{n}\left(\mu_{i k}\right)^{w}}
$$

5. Calculate the objective function iteration $\mathrm{t}(\mathrm{Pt})$ based on the equation (6).

$P_{t}=\sum_{i=1}^{n} \sum_{k=1}^{c}\left(\left[\sum_{j=1}^{m}\left(X_{i j}-V_{i j}\right)^{2}\right]\left(\mu_{i k}\right)^{w}\right)$

6. Calculating the matrix changes partitions based on the equation (7). 


$$
\begin{gathered}
\mu_{i k}=\frac{\left[\sum_{j=1}^{m}\left(X_{i j}-V_{i j}\right)^{2}\right]^{\frac{-1}{w-1}}}{\sum_{k=1}^{c}\left[\sum_{j=1}^{m}\left(X_{i j}-V_{i j}\right)^{2}\right]^{\frac{-1}{w-1}}} \\
\text { with } \mathrm{i}=1,2, \ldots, \mathrm{n} ; \text { and } \mathrm{k}=1,2, \ldots, \mathrm{c} .
\end{gathered}
$$

7. Checking the stopping conditions:

If Difference in value of the objective function at the iteration $\mathrm{t}(\mathrm{Pt})$ with the objective function at the iteration $\mathrm{t}-1$ (Pt-1) or less than the maximum iteration iteration then the stop condition which can be shown by the equation (8) and equation (9).

$$
\begin{aligned}
& (|\mathrm{Pt}-\mathrm{Pt}-1|<\xi) \\
& \text { ( }>\text { MaxIter) }
\end{aligned}
$$

Otherwise: continued iteration $\mathrm{t}=\mathrm{t}+1$ and repeat from step 4.

\section{a. Data and Variables}

\section{Research methods}

The data used in this study consisted of primary data in the form of patient data that is hidden identity, only loaded data 9 parameter fit was described on the variables and the way of analysis, namely: age, gender, race, blood pressure, blood sugar, cholesterol total, LDL (Low Density Lipoprotein), uric acid, BUN (Blood Urea Nitrogen), creatinine.

Data obtained as many as 239 of data (patient) by filing a data request to a hospital to have been hidden identity and conduct interviews with the neurologist for the validation of the data after the data obtained from the hospital. Such data will be used as training data and test data with a wide range of compositions in accordance with the test scenarios discussed in Chapter Results and Discussion.

Variables analyzed include levels of accuracy and processing time. Basically, analyze the results of clustering can be done by comparing the deviation of each cluster so that it looks homogeneity or heterogeneity of each cluster. This means that the smaller the deviation value, the better the resulting clusters, and this can be done by various methods. However, because this research cluster has resulted in rules that will be used in menginferensi input data so that it can be given outputs are classified into three levels of risk of stroke, the analysis of the strengths cluster can be seen from how much the level of accuracy / validity of classification status of the risk of stroke when to use each rules. So in this study, the analysis is done by comparing the accuracy of the results of the risk status by each rule generated each method.

Variable input, based on a literature review consists of nine parameters:
Age (U), Gender (JK), blood pressure (BP), Blood Sugar (KG), levels of total cholesterol (KT), Low Density Lipoprotein (LDL), Uric Acid (AU), BUN and creatinine $(\mathrm{K})$, with each having the interpretation of value (low, normal, high) as described in Review of Literature. While Outputs consist of 3 Status Risk: 0, 50,100 , where 0 means low risk, 50 medium risk and 100 high risk for stroke. Accuracy is obtained from the comparison of the risk status of the system outputs the actual risk status, risk status corresponding amount / correctly divided by the total number of test data, times $100 \%$.

Time is obtained from the length of the processing time of each method are immediately shown on the test.

\section{b. Problem Solution Framework}

The problems are expected to be completed in this study, based on the description in the Introduction, motivated by an effort to improve the accuracy of the Fuzzy Inference System. Fuzzy inference is used to resolve the question of classification or decision-making that has the problem of uncertainty by using the value of vagueness or ambiguity in reasoning. At the risk of stroke detection problem, the problem lies in the value of each parameter of the continuous and the boundary between one value with another small though biased provide different interpretations. So fuzzy is the right solution.

In a necessary inference rule of experts, but experts often can not express the whole knowledge explicitly, so that the results of the classification with fuzzy inference less than the maximum. Here we need a method that can generate rules based on the cases that have been resolved by experts with the knowledge and the results are correct. Based on preliminary research, there are two methods of generating rules for fuzzy inference Sugeno who have had the same accuracy is quite high and the difference in the results are not very significant, the problems of poor household classification. The method is substractive

Clustering and Fuzzy C-Means. It needs to be analyzed both methods to get the rule generation method is better for the detection of the risk of stroke.

In general, the solution of the problem described above is to take some of the data as training data, then the training data is grouped with the learning process using two kinds of methods, substractive Clustering and Fuzzy C-Means. From these groupings will emerge rules, where a rule is a bunch of data that has many similarities to the data value. The results were excluded from the process is a rule which may be the same or different. Each rule is then used to give a decision on the risk status of test data with Sugeno Fuzzy Inference order 1. The decisions by the two rules are then respectively compared with the actual decision of the experts, so we get the accuracy of each method of generating rules, In terms of processing time is also measured by the system so that it can be compared which method is time more quickly. 


\subsection{Design}

\subsubsection{Power Design Rules with substractive} Clustering

The design process of grouping data to generate rules with substractive clustering method shown in Figure 1.

The process of grouping data to find rules with substractive clustering method begins with the normalization of training data which is record data medic with 9 input parameters and outputs a value of risk status.

Furthermore sought early potential of each data point by calculating the distance between the data for the cluster center candidate. Having in mind the distance between the respective data, then set the value of $\mathrm{D}$ as a potential value of early and be checked repeatedly for to get the highest value $M$. The next step is the process of determining the center of the cluster, so we get the number of clusters formed and cluster centers selected. Then the value of the center cluster in normalized form is returned to its original shape before normalized, so the cluster center value is the true value. Furthermore sigma value is calculated for each attribute cluster center. The values are then used to find the value of the degree of membership of each data point. Last training data will be grouped into clusters that are formed based on the degree of membership of each point to the cluster center.

\subsubsection{Power Design Rules with Fuzzy C-Means}

The design process of grouping data to generate rules with fuzzy c-means shown in Figure 2. The process of clustering with fuzzy c-means is the process of training for training data for grouping data results were used for the generation of fuzzy rules using fuzzy c-means algorithm.

Fuzzy clustering process flow with c-means as shown in Figure 2 begins with the formation of the initial partition matrix $U$ to obtain the data to the cluster membership degree $\left(\mu_{\mathrm{ik})}\right.$ with the dimensions of the amount of data $x$ number of clusters.
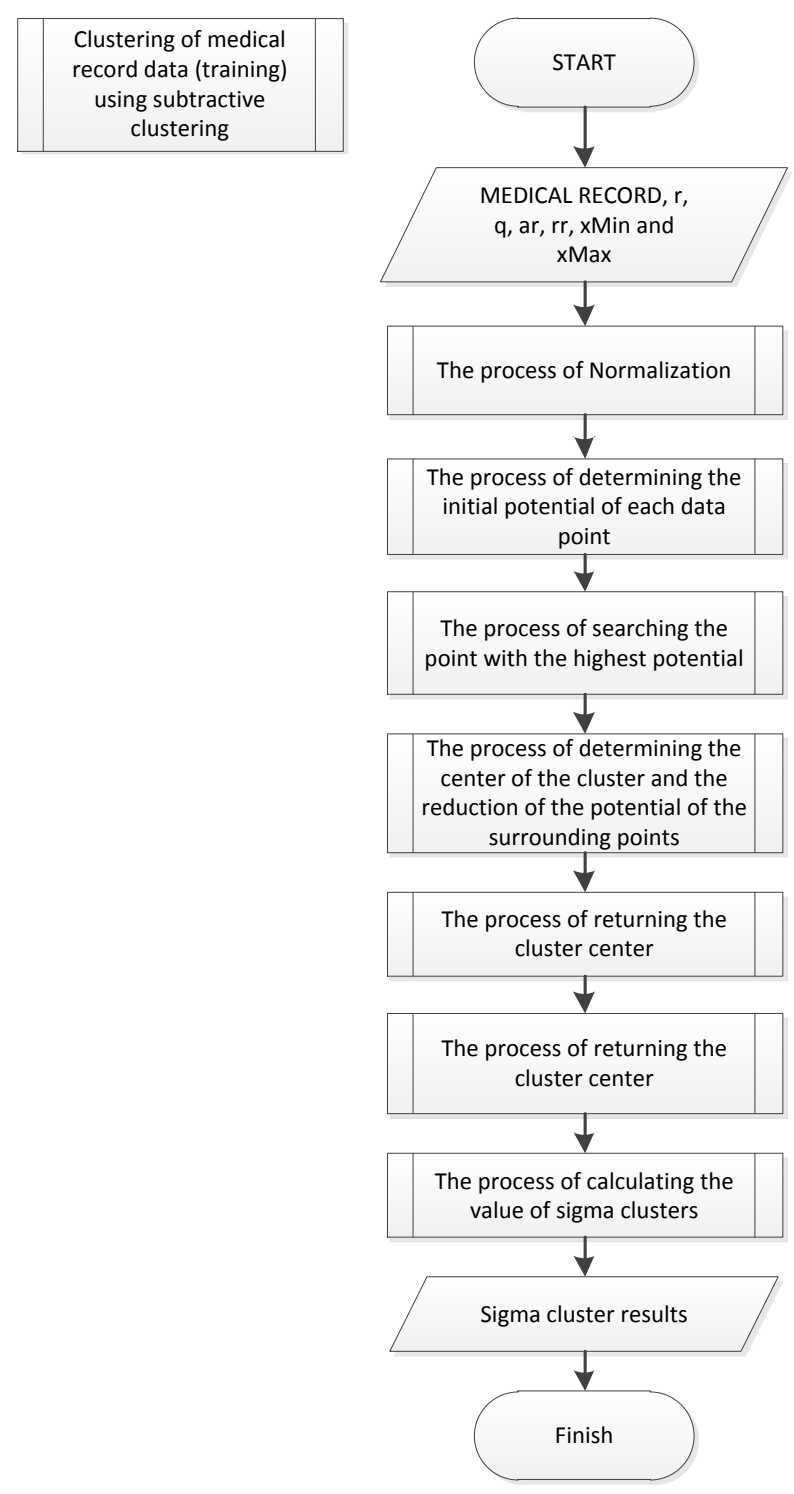

Figure 1 Flowchart grouping process for the generation of rule by substractive Clustering

5.3.2. Design of Generation Rules with Substractive Clustering

The design process of grouping data to generate rules with fuzzy c-means shown in Figure 2. The process of clustering with fuzzy c-means is the process of training for training data for grouping data results were used for the generation of fuzzy rules using fuzzy c-means algorithm. Fuzzy clustering process flow with $c$-means as shown in Figure 2 begins with the formation of the initial partition matrix $U$ to obtain the data to the cluster membership degree $\left(\mu_{\mathrm{ik}}\right.$ with the dimensions of the amount of data $\mathrm{x}$ number of clusters. 


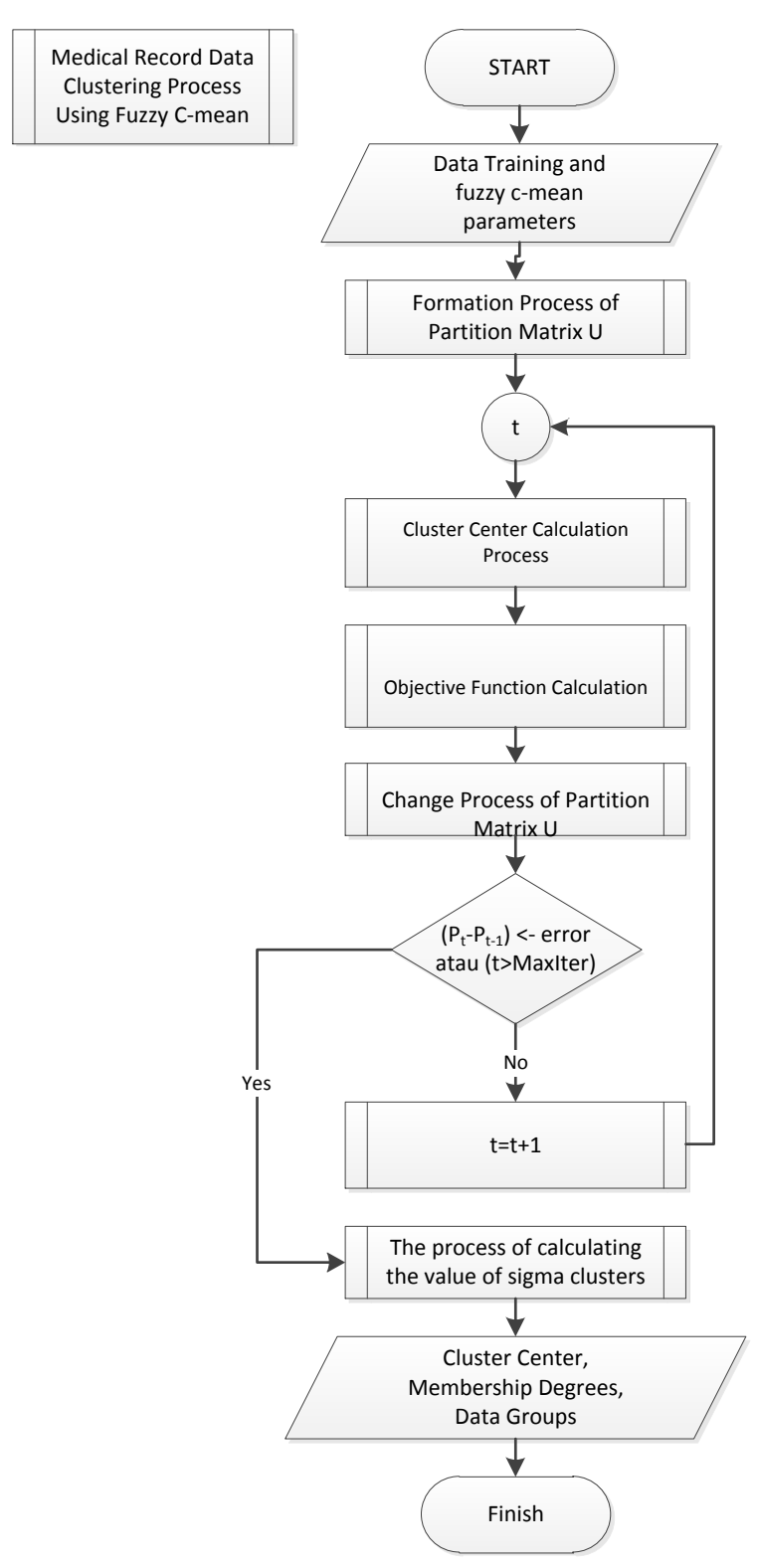

Figure 2 Flowchart process FCM

Next will be calculated cluster centers are then calculated the objective function. The objective function is used to calculate the difference between error and comparing it to a predetermined minimum error to provide the conditions to stop the iteration process of grouping data. The next step is calculating the partition matrix $U$ changes to improve the value of the degree of membership. Recently conducted grouping of data for classifying training data into clusters that are formed based on the degree of membership of each point to the cluster center.

\section{Results and Analysis}

\subsection{Rules Producedur Both Methods}

The resulting rules are tested with the testing rules that aims to determine whether the application has been implemented based on the design by the rule generation algorithm can provide the appropriate rules with expert knowledge. Testing

for FCM and substractive Clustering is done by running the program with variation determination rule generation variant limits and the number of clusters, performed 10 times to determine the stability of the results of different rules with given parameters or run several times. Then visits the accuracy of training data. While testing for substractive clustering is done by running the program with variant parameter variation limits, and fingers and reject ratio, then see how the influence of each parameter.

The parameter of question is a variant limits and the number of clusters. Testing was performed with 10 types of combinations limits and the number and variant parameters added to the reject ratio substractive method Clustering. Where the value of 0.0005 and 0.0004 variant being the number of clusters 3 and 4 on FCM, Election value limits the number of clusters of variants and after some trial and figures tersbeut that produces good results. Limitation of variance indicated limits on each cluster variant. If determined on the smaller value then the cluster is getting stronger or homogeneity level in one high cluster. The problem is if made very small, can happen a data difficulty to enter the cluster. The number of clusters to determine the desired cluster is formed, representing the number of rules. With tested the number of different clusters are expected to get the number of clusters that can provide the results of the rules that best suit the expert's knowledge. Because if it is made smaller it will cause less accurate rules, but if it is very big make the rules also less efficient.

Examples of the results obtained for testing the rules on FCM shown in Table 9.

Table 9 Examples of Rules of the generation method

\begin{tabular}{|c|c|c|c|}
\hline rule type & $\begin{array}{l}\text { Limitations } \\
\text { variant }\end{array}$ & Total Cluster & accuracy \\
\hline rule 1 & 0.00 & 3 & $33 \%$ \\
\hline \multicolumn{4}{|c|}{ 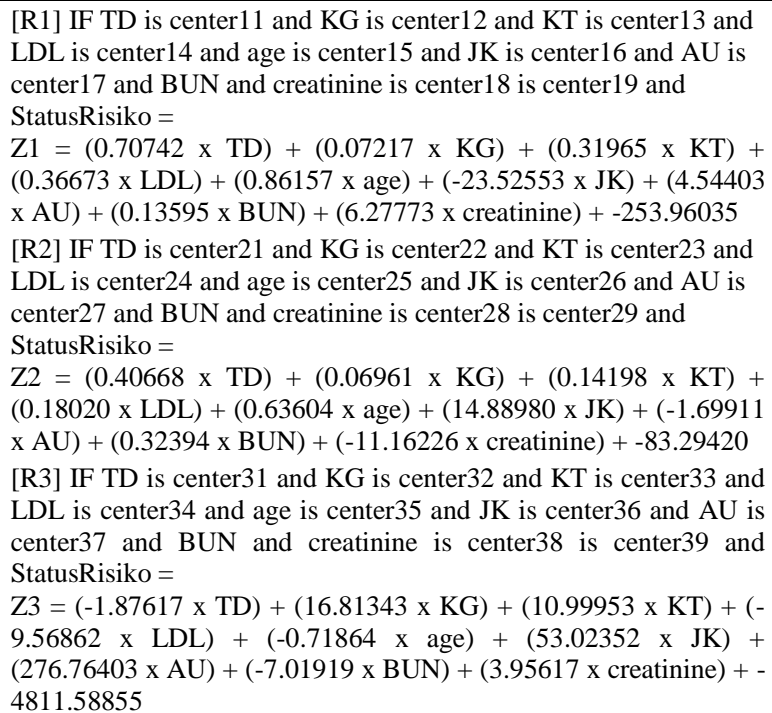 } \\
\hline
\end{tabular}

Under the rules of testing that has been done, it can be concluded that the rules which generated both a feasible method is used, due to the rules FCM compared with expert knowledge to get the value of an average accuracy of $87.33 \%$, and the resulting rules tend to be stable. And for substractive, visible from the constraints 
of a small variant. This is because, in the development of the iteration process to get the rules, with substractive method, must comply with the limits specified variants. While the variants show the distance between the data with other woods in one cluster. When the small variants means the degree of similarity in a large cluster. Limitation of 0.0004 variant was already small. So that applications built with substractive and FCM method in accordance with the design and implementation such as the one written in chapters 4 and 5 can be used to generate rules for detection for Sugeno fuzzy inference the risk of stroke.

\subsection{Comparison of Accuracy and Time Second Method}

To be able to compare the accuracy and processing time of both methods, then the comparison test of accuracy and and processing time is done. So that can be done comparison, which is better than both methods of generation of rules for the detection of risk of stroke, judging by the accuracy and processing time. In addition, with this test at the same time can know the influence of number and variation of training data to the accuracy results. Testing is done 24 times using all test data (139 data) to speed and accuracy. In the training process, using data train as much as 100 data and 80 data. Provided the data with the same amount will result in performing the training process with different data id, eg if the first 100 data will then be acted with id ranging from 1-100, for the second experiment starts id 11-110, Testing the amount of different trainer data is intended to determine the effect of the amount of training data on accuracy, both on substractive and FCM. The following test results are presented in Table 9.

Table 9. Comparison of average speed and accuracy of the method

\begin{tabular}{|c|c|c|c|c|}
\hline No. & $\begin{array}{l}\text { Amount } \\
\text { of data }\end{array}$ & Method & $\begin{array}{l}\text { Free-milli- } \\
\text { second } \\
\text { (rata2) }\end{array}$ & $\begin{array}{l}\text { Accuracy } \\
\text { (rata2) }\end{array}$ \\
\hline 1 & \multirow[t]{2}{*}{100} & subtractive & 33421.55 & $84.458 \%$ \\
\hline 2 & & $\mathrm{FCM}$ & 11550.58 & $75.248 \%$ \\
\hline 3 & \multirow[t]{2}{*}{80} & subtractive & 26123.60 & $69.990 \%$ \\
\hline 4 & & $\mathrm{FCM}$ & 14488.77 & $29.496 \%$ \\
\hline
\end{tabular}

Based on the results in Table 9, the first can be concluded that the amount of training data greatly affect the result accuracy. It is seen from an average of 100 training data, its accuracy is better than 80 training data, for both the FCM and substractive method.

At FCM even decreased very high. This is because the method of FCM in the process of determining initial cluster centers randomly and repeatedly repaired, unlike substractive already determine the center cluster with the highest compute density.

The second thing that can be analyzed is a comparison of the accuracy of the two methods. This analysis was based on the results with the best training data. So just to be analyzed in the training data 100 . On average, substractive gives better results than FCM with an average accuracy of $84.46 \%$, compared to $75.25 \%$. To substractive looks ever achieve $86.33 \%$ accuracy. However, the highest accuracy can not be used as a reference for both methods of training and there is a random element, so each time be run, the result is also a distinct possibility. But seeing an average 84.46 with a standard deviation of 0.9 , it can be said that the method substractive clustering, provides good results and stable accuracy, better than FCM method, for the detection of the risk of stroke by an average margin of $10.65 \%$ accuracy. A more detailed comparison is shown in Table 10.

Table 10 Comparison of the accuracy of the two methods in detail

\begin{tabular}{|l|r|l|}
\hline \multicolumn{2}{|c|}{ methods in detail } \\
examination & substractive & $F C M$ \\
\hline Tests 1 (first training data 100) & $84.17 \%$ & $84.89 \%$ \\
\hline $\begin{array}{l}\text { Tests 2 (100 second training data, the } \\
\text { last 10\% overlap with previous data) }\end{array}$ & $84.89 \%$ & $86.33 \%$ \\
\hline $\begin{array}{l}\text { Tests 3 (third training data 100, the } \\
\text { last 10\% overlap with previous data) }\end{array}$ & $85.61 \%$ & $50.36 \%$ \\
\hline $\begin{array}{l}\text { Testing 4 (fourth training data 100, } \\
\text { the last 10\% overlap with previous } \\
\text { data) }\end{array}$ & $84.89 \%$ & $72.66 \%$ \\
\hline $\begin{array}{l}\text { Testing 5 (fifth training data 100, the } \\
\text { last 10\% overlap with previous data) }\end{array}$ & $82.73 \%$ & $74.82 \%$ \\
\hline Average & $84.46 \%$ & $73.81 \%$ \\
\hline The average difference & \multicolumn{2}{|c|}{$10.65 \%$} \\
\hline \begin{tabular}{l|l|}
$|c|$ \\
standard deviation
\end{tabular} & 12.90 \\
\hline
\end{tabular}

The third analysis, the processing time. Based on Table 10 shows that the FCM provides rule generation processing time is faster. More details can be seen in Table 11 below.

Table 11. Comparison of two methods of processing time

\begin{tabular}{|l|r|r|r|}
\hline & \multicolumn{2}{|c|}{ time (ms) } & \multicolumn{1}{c|}{ Average } \\
\hline substractive & 33421.55 & 26123.6 & 29772.58 \\
\hline FCM & 11550.58 & 14488.77 & 13019.68 \\
\hline difference & 21870.97 & 11634.83 & 16752.9 \\
\hline
\end{tabular}

Substractive gives the average processing time $29772.58 \mathrm{~ms}$ or 29.77 seconds, while FCM 13019.68 ms or 13:02 seconds. There is a time difference of 16.75 seconds. But still there is a possibility in the methods substractive implementation is made more efficient coding, especially for some looping. Because of the implementation are made there looping made twice when in fact it can be quite a time.

From the analysis of the accuracy and the processing time it turns out the problem of the detection of the risk of stroke, substractive Clustering is superior in terms of accuracy, but the FCM is superior in terms of speed. So as to obtain better accuracy requires a longer time. In use we can determine whether more emphasis to high accuracy or fast time. The challenge ahead is to provide a method that better accuracy with faster time. Both accuracy and speed, the two methods could be 
developed by adding training data, or add a particular mathematical model or mengefisiensi program.

This study uses the same data to study Ahmad F Sholeh, which in the study for the detection of the risk of stroke using inference fuzzy without generating rules produce accuracy of $82.98 \%$. Substractive Clustering is superior, but the FCM accuracy underneath. So when the rules do specialists are already approaching the knowledge he has, then it can give a good accuracy. But the rule generation method can work smarter by raising rules in accordance with the expert knowledge and can give better results.

\section{Conclusion}

Generating rules on fuzzy inference so that computers can reason with knowledge as experts, can be done with substractive clustering method by searching normalized data first, then determining the initial potential of data points, looking for potency point that has the highest density, determining cluster center, restoring center cluster To the initial value, and group the data into the cluster by calculating sigma. While FCM is done by forming a matrix, determining the center of the cluster, calculating the objective function to meet the specified error limits, then grouping the data.

1. Substractive Clustering gives the average accuracy of $84.46 \%$, while $73.81 \%$ FCM, so substractive clustering provides better accuracy rate.

2. The average processing time for generating rules for substractive clustering is 29.77 seconds, while the FCM is 13:02 seconds. So that time, FCM faster.

3. In the detection problems of disease risk of stroke, a much-needed accuracy than the speed at which the difference is only tens of seconds, because the method substractive clustering better implemented on this issue.

4. Substractive clustering for the generation of rules on inference fuzzy proven to provide better results than the inference fuzzy without generating rules.

Suggestion :

1. Needs to be tested with more training data and determine what the optimum training data that can provide high accuracy.

2. Programming logic can be developed more efficient for both methods

3. Need to develop a mathematical model to study how humans can approach smarter so they can obtain better accuracy.

4. Needs to be studied, the advantages of FCM, in addition to the speed might be able to provide high accuracy for data with certain characteristics.

\section{References}

[1]. Abas, FS, K. Martinez. 2003. " Classification of Painting Cracks for Content-Based Analysis ". ECS, Soton
[2]. Arapoglou, Roi, Kostas Kolomvatsos and Stathes Hadjiefthymiades. 2010. " Buyer Agent Decision Process Based on Fuzzy Rules Automatic Generation Methods" .

[3]. Chiu, Stephen I." An Efficient Method for Extracting FuzzyClassification Rules from High-Dimensional Data

[4]. Jang, JSR, Sun, CT, Mizutani, E. 1997. " Neuro-Fuzzy and Soft Computing " . Prentice-Hall International, New Jersey.

[5]. Kusumadewi, $\mathrm{S}$ and Purnomo, H. 2010. " Application of Fuzzy Logic for Decision Support ". Yogyakarta .: Graha Science.

[6]. Kusrini. 2008. Application of Expert System menentuan User Certainty Factor with Quantitative Methods Questions, Andi, Yogyakarta.

[7]. Sayekti, Ely Ratna. 201 4. " Implementation Algorithm Fuzzy C-Means Clustering for Fuzzy Rule Generation At Risk Level Grouping Breast Cancer ". Brawijaya University. Malang .

[8]. Sholeh, Ahmad Fashel. 2012. " Application of Decision Support for Early Detection of Disease Risk Stroke Using Logic Fuzzy Mamdani: Case Studies from RS XYZ ". Essay. Higher Institute of Ten November .Surabaya.

[9]. Widiyanto, Agung Putra. 2013. " Implementation of Subtractive Clustering Algorithm for Fuzzy Rule Generation In Recommendation Scholarship " . Brawijaya University, Malang.

[10]. Yastroki. 2011. " Overview of Stroke ". Specialist Doctors Association of Indonesian Neurosurgery and Stroke Foundation of Indonesia 